and external laser beams. Consequently, the polarisation and intensity of light emitted by polariton lasers can be switched from one value to another within several tens of picoseconds ${ }^{9}$. This controllability and fast response make polariton lasers promising for applications in optical integrated circuits at the interface between electronics and optical communication devices. Another application area that remains to be explored is terahertz frequency generation by polariton condensates $^{10}$. Given the high demand for compact and reliable sources of coherent terahertz radiation, bosonic cascade lasers based on excitons or exciton polaritons offer a valuable alternative to quantum cascade lasers based on electronic transitions in semiconductor superlattices. Potentially, they could operate at room temperature, emit terahertz light in the vertical direction (that is, normal to the plane of the structure) and be as small as any vertical-cavity surface-emitting laser.

The next milestone on the path to developing a truly practical polariton laser will be the demonstration of roomtemperature operation under electrical injection. Until recently, GaN-based microcavities had been considered as most the promising means to meet this objective given that excitons in $\mathrm{GaN}$ are stable at room temperature ${ }^{6}$. However, one should not forget GaAs-based microcavities, which are worthy of further investigation. The strong coupling to microcavity modes stabilizes excitons in GaAs, which is why they might survive up to room temperature in carefully designed structures. I expect very significant further advances in the area of polaritonics in the coming years, if not months.
Alexey Kavokin is at the Physics and Astronomy School, University of Southampton, Highfield, Southampton, SO171BJ, UK and the Spin Optics Laboratory, State University of St. Petersburg, 1, Ulianovskaya, 198504, Russia.

e-mail:alexey@phys.soton.ac.uk

References

1. Einstein, A. Verh. Deutsch. Phys. Gesell. 18 318-323 (1916).

2. Coldren, L. A. \& Corzine, S. W. Diode Lasers and Photonic Integrated Circuits (Wiley, 1995).

3. Imamoğlu, A., Ram, R. J., Pau, S. \& Yamamoto, Y. Phys. Rev. A 53, 4250-4253 (1996).

4. Bloch, I., Hänsch, T. W. \& Esslinger, T. Phys. Rev. Lett. 82, 3008-3011 (1999).

5. Christopoulos, S. et al. Phys. Rev. Lett. 98, 126405 (2007).

6. Schneider, C. et al. Nature 497, 348-352 (2013).

. Bhattacharya, P., Xiao, B., Das, A., Bhowmick, S. \& Heo, J. Phys Rev. Lett. 110, 206403 (2013).

8. Rubo, Y. G., Kavokin, A. V. \& Shelykh, I. A. Phys. Lett. A 358, 227-230 (2006).

9. Amo, A. et al. Nature Photon. 4, 361-366 (2010)

10. Liew, T. C. H. et al. Phys. Rev. Lett. 110, 047402 (2013).

\title{
OPTICS
}

\section{The flame lens}

Scientists in South Africa have developed a hot-gas lens that possesses improved optical capabilities and a damage threshold that is estimated to be vastly superior to that of conventional glass optics. Once optimized, such lenses may be useful for focusing ultra-intense laser beams such as those used in X-ray lasers, laser-driven accelerators and laser fusion experiments.

The 'flame lens' is the inspiration of Max Michaelis, Andrew Forbes and co-workers at the University of KwaZulu-Natal and the National Laser Centre in South Africa (Nat. Commun. 4, 1869; 2013). "Our lens can transmit beams whose intensities are two orders of magnitude higher than the maximum intensity that solid-state lenses can transmit without sustaining damage," commented Forbes. "Even if breakdown were to occur, the lens repairs instantaneously, unlike solid-state optics, which are either permanently impaired or must be left to cool for hours."

The idea of using a hot metal tube to create a temperature gradient and thus a lens-like refractive index profile in a gas has been around for some time. Bell Laboratories in the USA investigated the idea in the 1960s not long after the development of the first lasers. Michaelis has been doggedly pursuing the concept and trying to make it practical for the past 25 years.

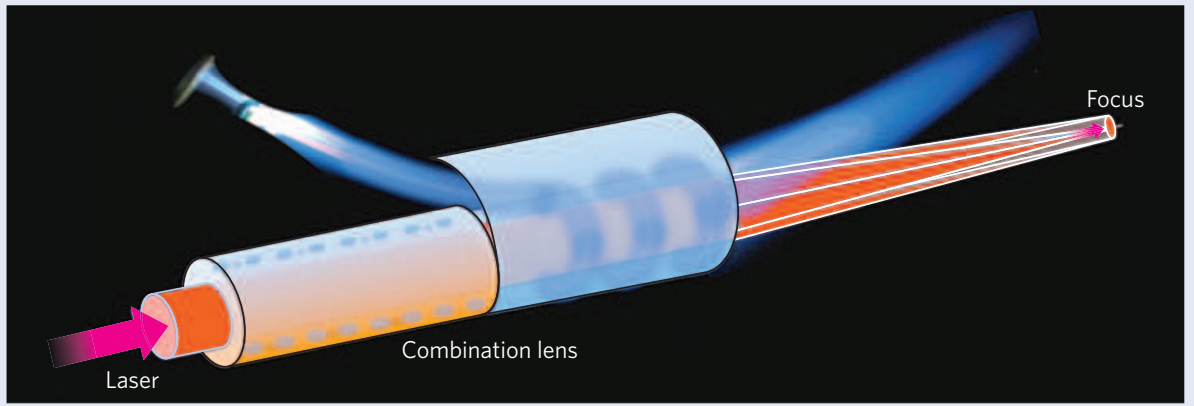

Early designs were plagued by severe limitations in terms of their large size, high complexity and weak focusing. "These early tube gas-lens designs suffered from three fundamental problems - their apertures were small (of the order of $7 \mathrm{~mm}$ ), their focal lengths were very long $(2.5 \mathrm{~m}$ to $10 \mathrm{~m}$ ) and they required complicated ancillary apparatus," explained Forbes. "These early lenses were usually of the order of a metre in length, and were thus long and bulky."

To address these issues, the South African team designed a composite gas lens that consists of two parts. The first stage is a $50-\mathrm{mm}$-long metal-tube gas lens with a $10-\mathrm{mm}$-diameter aperture that is heated from below and refracts the outer rays of a light beam. The second stage is a shorter tube, $25 \mathrm{~mm}$ in length; it contains a spiral flame that mainly acts on the inner rays. The stainless-steel tubes of both lenses are heated to around $400{ }^{\circ} \mathrm{C}$ so that they become red hot. The result is a flame lens, which brings light to a sharp focus and is more compact and has a focusing power that is four times stronger per unit length than earlier gas-lens designs.

The team has used a prototype flame lens with a focal length of about $2 \mathrm{~m}$ in proof-of-principle experiments that include focusing of high-intensity light, imaging of highly chromatic sources and drilling plastic with high-energy pulses. The team is now using aerodynamic theory to optimize the lens structure and further improve its performance. "We predict that this will remove all the aberrations bar astigmatism, bringing the flame lens into a similar quality realm as conventional lenses," said Forbes. "One could also consider operating the lens at higher pressures or with different gases to decrease the focal length."

OLIVER GRAYDON 